\title{
Correspondences between parameters in a reaction-diffusion model and connexin functions during zebrafish stripe formation
}

\section{Akiko Nakamasu ( $\square$ nakamasu@kumamoto-u.ac.jp )}

Kumamoto Univ.

\section{Article}

Keywords: Pattern formation, Turing pattern, mathematical model, Reaction-diffusion system

Posted Date: July 14th, 2021

DOI: https://doi.org/10.21203/rs.3.rs-658862/v2

License: (c) (1) This work is licensed under a Creative Commons Attribution 4.0 International License.

Read Full License

Version of Record: A version of this preprint was published at Frontiers in Physics on January 18th, 2022. See the published version at https://doi.org/10.3389/fphy.2021.805659. 
1 Title; Correspondences between parameters in a reaction-diffusion

2 model and connexin functions during zebrafish stripe formation.

3 Running Head; Link between experimental and theoretical Turing patterns

4 Akiko Nakamasu*

5 International Research Organization for Advanced Science and Technologies, Kumamoto

6 University, 2-39-1 Kurokami, Chuo-Ku, Kumamoto, 860-8555 Japan

$7 \quad$ * Corresponding author

$8 \quad$ Email: nakamasu@kumamoto-u.ac.jp

$9 \quad$ Keywords

10 Pattern formation, Turing pattern, Mathematical model, Reaction-diffusion system. 


\section{Statements}

manuscript nor the study violates any of these, as follow. Data can be provided on request.

4 This research was supported by Grant-in-Aid for Scientific Research on Innovative Areas

5 (Japan Society for the Promotion of Science), Periodicity, and its modulation in plants.

6 No.20H05421. There are no conflicts of interest to declare. This study is not related to the

7 ethics approval and patient consent statement. Then the manuscript including pictures from

8 Usui et al. (2019) Development 146, dev181065 in Fig.4 and Fig. 6. The use of the pictures

9 was permitted by the author M. Watanabe. 


\section{$1 \quad$ Key Findings}

2

3

4

5

6

7

8

9

10

- An experimentally derived mathematical model could be applied to experimental results published since the original development of the model.

- The terms of the mathematical model could be connected with the functions of molecular channels on different cells.

- The model used partial differential equations (PDEs) enabled numerical and mathematical analyses of characteristics observed in the experiments.

- Further improved PDE model including nonlinear reaction terms, enabled the consideration of the behavior of components. 


\section{Abstract}

Different diffusivities among interacting substances actualize the potential

3 instability of a system. When these elicited instabilities manifested as forms of spatial

4 periodicity, they are called Turing patterns. Simulations using general reaction-diffusion

5 (RD) models have demonstrated that pigment patterns on the body trunk of growing fish

6 follow a Turing pattern. Laser ablation experiments performed on zebrafish revealed

7 apparent interactions among pigment cells, which allowed for a three-components RD

8 model to be derived. However, the underlying molecular mechanisms responsible for

9 Turing pattern formation in this system had been remained unknown. A zebrafish mutant

10 with a spotted pattern was found to have a defect in Connexin41.8 (Cx41.8) which,

11 together with Cx39.4, exists in pigment cells and controls pattern formations. Here,

12 molecular-level evidence derived from connexin analyses was linked to the interactions

13 among pigment cells described in previous RD modeling. Channels on pigment cells were

14 generalized as "gates," and the effects of respective gates were deduced. The model used

15 partial differential equations (PDEs) enabled numerical and mathematical analyses of

16 characteristics observed in the experiments. Furthermore, the improved PDE model

17 including nonlinear reaction terms enabled the consideration of the behavior of

18 components. 


\section{Introduction}

2

3

4

5

6

7

8

9

10 formation studies. ${ }^{3,4}$ Zebrafish have a pattern of stripes on their body and fins (Fig. 1A).

11 The pattern is generated by three types of pigment cells: complementarily distributed black melanophores and yellow xanthophores (Fig. 1C), plus iridescent iridophores. Numerous zebrafish pigment-pattern mutants were artificially generated ${ }^{6}$ and the corresponding genes

14 were identified. One of the most important mutants is leopard, which produce a spotted

In 1952, Alan Turing postulated that two substrates interacting with each other

show instability when they diffuse at different speeds. He explained this diffusion-driven

instability by utilizing a linear reaction-diffusion (RD) model. This model demonstrated that

spatial inhomogeneity (i.e., Turing pattern) could be generated by such conditions. ${ }^{1}$ This

relationship is known to generate patterns, though the components remain to be explored.

More than two decades ago, Kondo and Asai (1995) reported that pigment

stripes on the bodies of growing marine angel fish behaved as a Turing pattern. ${ }^{2}$ Research

focus then shifted mainly to zebrafish (Danio rerio) as a model organism for pattern

were identified. One of the most important mutants is leopard, which produce a spotted

pattern, that is representative of Turing patterns (Fig. 1B). Watanabe et al. (2006) identified

connexin41.8 (cx41.8) as the gene responsible for the leopard mutation. ${ }^{7}$ Besides Cx41.8,

other connexins such as Cx39.4 exist in pigment cells and affect pigment-pattern formation.

Six connexins form a hemi-channel (or "connexon"), which connects intracellular and 
1 extracellular spaces (Fig. 1D). Docking of two hemi-channels from adjacent cells give rise

2 to a gap junction, which mediates intercellular signal transfer. ${ }^{8}$ The minimal connexin

3 network required to originate a striped pattern was recently revealed by regulating connexin

4 expression in each pigment cell. ${ }^{9}$ Therefore, these channels are important for pigment-

5 pattern formation. Interactions among pigment cells and their molecular mechanisms involved in

7 pattern formation are summarized in Volkening $(2020) \cdot{ }^{10}$ However, the molecular

8 mechanisms leading to Turing instability have remain mostly unresolved. Mosaic fish

9 experiments have indicated that both leopard/cx41.8 and jaguar/obelix/kcnj13 genes are

10 required for segregation of melanophores and xanthophores. Such segregation was

11 proposed to involve local interactions between adjacent pigment cells. ${ }^{11}$ Xanthophore

12 ablation using a temperature-sensitive csf1ra allele led to the gradual death of

13 melanophores in both the body trunk and fins of adult fish. ${ }^{12}$ Accordingly, melanophore

14 survival requires continuous signaling from xanthophores. Laser ablation of stripe and inter-

15 stripe areas has revealed the mutual interactions between melanophores and

16 xanthophores. ${ }^{13}$ The interactions comply with the requirements for Turing pattern formation

17 (Fig. 1E). Specifically, both types of pigment cells activate their own types at a single-cell

18 distance (short range) by inhibitions of other types, but then inhibit their own types beyond 
1 the width of the stripe (long range). The difference in reaction distances achieves the "local

2 activation and lateral inhibition" condition needed for pattern formations. ${ }^{14}$ It should be

3 noted, however, that the distinction between iridophores and xanthophores was sometimes

4 unclear in those experiments.

6 a highly diffusible molecule (i.e., long-range factor) and two cells (regarded as short-range

7 factors with low diffusivity) was constructed. ${ }^{13}$ This three-component RD model, with its

8 linear reaction terms and upper and lower limits, described the apparent interactions

9 obtained experimentally. Then the different diffusibilities and the interactions in the model

10 achieved diffusion-driven instability (Turing instability). projections. ${ }^{15}$ The interaction mediated by gap junctions on the tip of the projection was

13 considered to be a long-range effect observed in the previous experiments. ${ }^{16}$ The

14 researchers mentioned the possibility that the pattern formation might not require actual

15 diffusion. Later a Turing model based on an integral kernel was suggested, ${ }^{17}$ though the

16 link between parameter and molecular function was ambiguous. Most other models for the

17 pigment-pattern formation are based on interactions at a cellular level. These models

18 implemented different effects depending on the distance from each pigment cell. ${ }^{18,}$ 19, 20 
1 Several attempts were made to explain the observed patterns in zebrafish mutants by a

2 general Turing model, ${ }^{21,}{ }^{22}$ however, they were not supported experimentally, even though

3 there are several paths to cause the expected pattern changes in mutants. Here, the interactions in a three-component model, including a highly diffusible

5 factor, were developed to attempt to link the molecular functions of connexins in zebrafish.

6 Channels thought to be important for the pattern formation were generalized as "gates" of

7 pigment cells. These gates enable transport of the diffusible molecule across the

8 membrane. The parameters affected by each gate were deduced, then the effects on

9 pattern-selection and -size, were analyzed. Finally, the model was improved to an

10 analogous model with nonlinear terms. These models together enabled reasonable

11 explanations of detailed behavior of the components that related to the pattern formation. 


\section{$1 \quad$ Results}

\section{Linear reaction-diffusion model for pigment-pattern formation}

interactions between two types of pigment cells were necessary to generate Turing

7 Nakamasu et al. (2009), ${ }^{13}$ although the details of the relationship between the experimental results and the model were not described. This model was based on the following set of RD equations:

$$
\left\{\begin{array}{l}
\frac{\partial u}{\partial t}=D_{u} \nabla^{2} u+f(v, w)-d_{u} u \\
\frac{\partial v}{\partial t}=D_{v} \nabla^{2} v+g(u, w)-d_{v} v \\
\frac{\partial w}{\partial t}=D_{w} \nabla^{2} w+h(u, v)-d_{w} w
\end{array}\right.
$$

12 (viability). Numerical simulation of this model resulted in a Turing pattern in which $u$ and $v$

13 were distributed with antiphase while a concentration of third factor $W(w)$, presented

14 peaks synchronized with $u$ (Fig. 2). It should be noted that cell divisions of differentiated 
1 Therefore, the number of melanophores is changed by 1) supply of new cells from

2 randomly scattered precursor cells, 2) death of existing pigment cells, or 3) migration from

3 a position close to the skin surface. ${ }^{23}$ In the case of melanophores, it is known that cell

4 movements and cell deaths are complementary to each other. ${ }^{24}$ Even though they are

5 inhibited, xanthophores are found in the stripe region, where they exist with a pale color. ${ }^{25}$,

$6 \quad{ }^{26,27}$ Xanthophores do not move actively in vivo, as may be the case for iridophores. As

7 detailed in Fig. 1F, motilities of the cells were approximated by small diffusion coefficients

$8 \quad\left(D_{u}\right.$ and $\left.D_{v}\right)$. The rapidly diffusing factor $W$ was assigned a large diffusion coefficient

$9 \quad\left(D_{w}\right)$. The parameters in the reaction formulae were positive constants as shown in

11 Table 1. Each formula was composed of a set of linear terms with upper and lower limits as

12 follows:

$$
\left\{\begin{array}{c}
f(v, w)=\left(\begin{array}{c}
0 ;-i_{u v} v-i_{u w} w+s_{u}<0 \\
-i_{u v} v-i_{u w} w+s_{u} ; 0<-i_{u v} v-i_{u w} w+s_{u}<f_{\max } \\
f_{\max } ;-i_{u v} v-i_{u w} w+s_{u}>l_{\max } \\
0 ;-i_{v u} u+p_{v w} w+s_{v}<0
\end{array}\right) \\
g(u, w)=\left(\begin{array}{c}
-i_{v u} u+p_{v w} w+s_{v} ; 0<-i_{v u} u+p_{v w} w+s_{v}<g_{\max } \\
g_{\max } ;-i_{v u} u+p_{v w} w+s_{v}>m_{\max } \\
0 ; p_{w u} u-c_{w v} v+s_{w}<0
\end{array}\right) \\
h(u, v)=\left(\begin{array}{c}
p_{w u} u-c_{w v} v+s_{w} ; 0<p_{w u} u-c_{w v} v+s_{w}<h_{\max } \\
c_{\max } ; p_{w u} u-c_{w v} v+s_{w}>n_{\max }
\end{array}\right)
\end{array}\right.
$$

14 assumed to be produced by $U\left(p_{w u}\right)$ and consumed by $V\left(-c_{w v}\right)$. Accordingly, $W$ was 
1 assumed to inhibit pigment cells of the producer $\left(-i_{u w}\right)$ and produce (or preserve) the

2 consumer $\left(p_{v w}\right)$. The self-coupling parameters $-d_{u},-d_{v},-d_{w}$ corresponded to

3 degradation (or death) coefficients, whereas s's represented constants related to supply

4 (also called "support sustainability") of each component. The producer $U$ activated $V$, but

5 then inhibited itself at long range via $W$. By consuming $W, V$ also indirectly inhibited itself,

6 but then was activating $U$ by double inhibition at long range. As a result, $U$ and $V$

7 exhibited no difference in apparent interactions, making it difficult to identify which factor

8 corresponded to which cell type. Besides melanophores, xanthophores also showed self-

9 inhibition at long range. In laser ablation experiments, melanophore elimination in adjacent

10 stripes caused pale-colored xanthophores in an inter-stripe region. Therefore, the pale

11 color reflects xanthophore inhibition even without a change in cell number.

13 to a combination of two-variable systems as follows.

$$
\left\{\begin{array}{l}
\frac{\partial x}{\partial t}=f_{x} x+f_{y} y+D_{x} \nabla^{2} x \\
\frac{\partial y}{\partial t}=g_{x} x+g_{y} y+D_{y} \nabla^{2} y
\end{array}\right.
$$
inhibitor type (Fig. 3A) or activator-substrate-depletion type (Fig. 3B), characterized by 
1 different signs of the parameters. ${ }^{28,29}$ Both conditions were included in the three-component

2 model (Fig. 3C). Therefore, each parameter in the model can be regarded as part of a two-

3 variable system. The first and second reaction formulae (2) include mutual inhibitions

$4\left(-i_{u v} v,-i_{v u} u\right)$, each of which corresponds to the respective self-activation $\left(f_{x}>0\right.$ for $\left.x\right)$,

5 though it cannot be realized without each partner. Recent experiments revealed that

6 xanthophores are generated from division of other xanthophores. ${ }^{27}$ Therefore, at least one

7 of self-reaction parameters; $-d$ for $u$ or $v$ in (2), cannot be a degradation coefficient, i.e.,

8 it might be a non-negative parameter.

10 Effects of the parameters on the component proportion and the characteristic

11 wavelength of a pattern.

13 changes in the types and sizes of patterns. The former is defined by pattern selection, ${ }^{30,31}$

14 and manifests as a general variation of the Turing pattern from spots, to stripes to reverse

15 spots. The latter is dictated by the characteristic wavelength of the pattern. ${ }^{32}$ The following

16 sections analytically describe the effect of parameters in the model (1)-(2) on these

17 characteristics. 


\section{Effects of parameter on pattern size} given by the following equations:

$$
\frac{2 \pi}{k_{\max }}=\sqrt{\frac{\sqrt{D_{x} D_{y}}\left(D_{y}-D_{x}\right)}{\left(D_{x}+D_{y}\right) \sqrt{-f_{y} g_{x}}-\sqrt{D_{x} D_{y}}\left(f_{x}-g_{y}\right)}}
$$

parameter in the model can be regarded as a two-variable system (Fig. 3C). The mutual

7 inhibitions $\left(-i_{u v} v,-i_{v u} u\right)$ correspond to the self-activation, though they are inversely

8 related, i.e., the sign of the parameters is different from $f_{x}$ in two-variable systems. The

9 increase in the absolute values of the parameters reinforces the self-activation. At the same

10 time, the effects of $-d_{u}$ and $-d_{v}$ are opposite of $f_{x}$. The effect on pattern size when the

11 absolute value of each parameter is decreased (i.e., the decline of each interaction) is

12 shown in Fig. 2D and indicates correspondence with two-variable systems (Fig. 3A, B).

\section{Effects of parameter on pattern selection} In zebrafish, pattern selection is determined mainly by the proportion of two 
1 equilibrium from the limits of the reaction terms provides an index for pattern selection, ${ }^{31}$ as

2 that is the origin of diffusion-driven instability.

4 decrease in each absolute value (i.e., the decline of each interaction) of the parameter with

5 a positive effect decreases the population volume of respective cells, then that of the

6 parameter with a negative effect increase the population of the respective cells. Here, the

7 component $W$ with high diffusivity represses $u$ and promotes $v$, therefore, the decrease

8 in the positive parameter in the differential equations of $w$ increases $u$ and decreases $v$

9 respectively. The opposite can apply to negative parameters. From this aspect, however, it

10 is difficult to refer about the combined effect of parameters with different signs.

Correspondence between the mathematical model and connexin defects in zebrafish estimated from molecular function

Next, correspondence between this model and molecular functions was assumed (Fig. 1G). In zebrafish, leopard mutants are known to have an aberrant pigment

16 pattern, whereby stripes are changed to $\operatorname{spots}^{33}$ (Fig. 1A, B). The gene responsible for the

17 leopard mutation is a connexin $c x 41.8$, which encodes a four trans-membrane connexin 
1 protein. Additionally, mutation of connexin cx39.4 results in wavy stripes. Recent analyses

2 of connexin activity have revealed different functions associated with hemi-channels and

3 gap junctions. ${ }^{34,35}$ Hemi-channels are open to the extracellular environment, whereas gap

4 junctions form connections between cells to allow the exchange of small molecules (Fig.

5 1D). Accordingly, connexins may be involved in both long- and short-range interactions.

6 These channels may function as gates for the transport of molecule $W$ across the

7 membrane (Fig. 1G). Accordingly, producer $U$ produces $W$, which then diffuses outside

8 the cell into the extracellular space via some kind of gate. Therefore, gate defects affect

9 survival of the producer by preventing release of harmful $W$ (i. e., $d_{u}$ of $-d_{u}$ is

10 increased). I considered two other possibilities, i.e., the effect of gate defect on $D_{w}$ and/or

$11 i_{u w}$. If $D_{w}$ was changed, $W$ outside of the cells will also be affected. A $u$-dependent

12 decrease in $D_{w}$ might be more appropriate for the assumption of enclosed $W$. Though, it

13 will give difficulties in the analytical approach and both will finally result in an increase in the

14 death of $U$. Then the degree of harmful effect on $U$ by same concentration of $W$ would not

15 change. Therefore, $D_{w}$ and $i_{u w}$ would not be affected. $w$ had peaks with the peaks of

16 producer $U$ and gates on cells assumed passive effects on $W$ movements. This explain

17 why $p_{w u}$ might not be affected, at least directly. Consumer $V$ was assumed to incorporate

18 beneficial $W$ into the cell across the gates then to consumes it, indicating that the gate 
1 defects will decrease the parameters $c_{v w}$ of $-c_{v w}$ and $p_{w v}$. Both parameters were

2 assumed to be related to intracellular events, therefore, higher $w$ was needed for the

3 same rate of consumption and $V$ production compared with the case of intact hemi-

4 channels. At the same time, parameters for mutual inhibitions $i_{u v}$ and $i_{v u}$ are decreased

5 by the leopard mutation, ${ }^{36}$ through gap junctions (combination of gates) at short range.

6 They would be simultaneously affected linking with the hemi-channel defect on the

7 corresponding cell. In Fig.1G, these parameters linked to different gates are indicated by

8 different hatchings.

10 Comparisons of results obtained by simulation and experiments. First, the independent effects of the gate on each cell were analyzed numerically.

12 When an arbitrary stripe is set as a starting point, only gates that opened to the outside on

$13 U$ and $V$ cells were removed along the $x$ - and y-axes. Numerical simulation of this model

14 yielded a spot pattern of $u$ in the case where gates on $U$ cells had defects, as expected

15 by the sing of the parameter (Fig. 4A). Reversed $u$ spots were yielded in $V$ cell defects

16 (Fig. 4B), though the change was not strong, because it included opposite effects on $V$

17 volume. Pattern simplicity score (PSS) increased in both cases, then overall color tones 
1 (OCT) were decreased and increased by respective defects. That is, the asymmetry of

2 changes in pattern selection can be observed by the removals of gates on respective cell

3 types. Defects on short-range inhibition did not have drastic effects on pattern selection,

5 though the pattern did finally disappear (Fig. 4C). On the 2D plane, the stripe region was

6 recessive together with the defect in short-range effects by gap-junctions (Fig. 4D-H, J),

7 though the tendency to shift the pattern selection was not changed. In Fig. 4I, short-range

8 effects were simultaneously decreased by respective $x$ or $y$ values that linked with $U$ or

$9 \quad V$ defects, as shown in right panel in Fig. 4J. This also kept the same tendency to shift the

10 pattern selection with individual cases. The results mostly consisted with the positive effects of connexin additions to

12 WKO that increased the respective pigment cells. ${ }^{9}$ However, experimental eliminations of

13 gate on each wild-type pigment cell led to an increase in the rate of respective pigment

14 cells; melanophore defects generated net patterns of melanophores, while the xanthophore

15 defects resulted in dot patterns of melanophores (i.e., net patterns of

16 xanthophore).Therefore, the simulation results were partly inconsistent with experimental

17 results in Usui et al. (2019), ${ }^{9}$ in which the effects of connexins on each or both pigment 18 cells were investigated in detail (Fig. 4K). 
2 type of connexin could be considered to have different strengths of the (hemi-) channel

3 functions on the two types of cells. Deduced patterns of respective transgenic fishes were

4 indicated by small letters on the phase plane in Fig. 4l. Even though the differences in the

5 strengths of channels were taken account, the removal of hemi-channels on wild-type

6 xanthophores tended to increase the population of xanthophore then a faint increase in

7 melanophores was brought in the case of connexin on melanophores (Fig. $4 \mathrm{~K}$ ). If cell $U$ is

8 a melanophore, other than the gray-framed patterns shown in Fig. $4 \mathrm{~K}$ did not seem to

9 correspond to Fig $4 \mathrm{I}$, though all of the experimentally obtained patterns are existed in the

10 simulation. As describe more distinctly, some shuffles can give a strong correspondence

11 with Fig. 4I (Fig. 6). From the analyses of wavelength mentioned above, defects to the gates on cell $U$

13 (increasing in $d_{u}$ of $-d_{u}$ ) or cell $V$ (decreasing in $p_{v w}$ and $c_{w v}$ ) caused a decrease or an

14 increase in pattern size, respectively. In numerical simulations, the pattern size tended to

15 be decreased and increased by the hemi-channel defects on both $U$ and $V$ cells, as

16 expected (Fig. 3A, B). The characteristic thinning of $u$ stripes and widening of $v$ inter-

17 stripes were observed in the simulation of $U$-cell defects, though it was not explained by

18 the analyses. From the characteristic thinning of the $u$ stripe, each cell corresponding to 
1 one of two short-range factors may be deduced. However, the thinning was observed on

2 melanophore stripe, in the case of defect in xanthophore. Inconsistent with the $U$ defect,

3 melanophore defect tented to result in wide melanophore stripes in the experiment.

\section{$5 \quad$ Non-linearization of the model}

6

To describe the behavior of the components in the system in greater detail, the

7 model was non-linearized. As an example, we devised a model with nonlinear terms as

8 shown in (5), though qualitative relationships were not changed from the model shown in

9 Fig $1 \mathrm{~F}$.

$$
\left\{\begin{array}{c}
\frac{\partial u}{\partial t}=D_{u} \nabla^{2} u-i_{u v} u v-i_{u w} u w+d_{u} u+\frac{s_{u}}{1+u+v} \\
\frac{\partial v}{\partial t}=D_{v} \nabla^{2} v-i_{v u} v u+p_{v w} v w-d_{v} v+\frac{s_{v}}{1+u+v} \\
\frac{\partial w}{\partial t}=D_{w} \nabla^{2} w+p_{w u} u-c_{w v} w v-d_{w} w+s_{w}
\end{array}\right.
$$

This model, using nonlinear RD equations, also generated Turing patterns (Fig.

11 5). Considering interactions between different types of cells and between cells and

12 molecules, inhibitions were given by multiplication terms (e.g., the inhibition of $U$ by $W$

13 was described as $u w$, and so on). These multiple terms are based on the description of

14 second-order reaction in chemical kinetic equation or dimer reactions by Meinhardt 
1 (1982), ${ }^{37}$ it enabled only limited reaction by the contacts between the components. $W$ was

2 needed for maintenance of cell $V$ so this reaction was also given by a multiplication term

3 of their volume. On the other hand, production of $W$ by $U$ will only occur $u$-dependently,

4 and degradation (and deaths) will also be $w$-dependent. Therefore, those terms are linearly

5 related to each component. These non-linearizations can identify the actions on an existing

6 cell or a newly differentiating cell. Then new generations of pigment cells occur only with

7 eliminations of existing cells. ${ }^{38}$ Therefore, mature cells would inhibit the generation of cells.

8 The sign of $d_{u}$ seemed prefer to be positive for the start point of pattern selection

9 corresponded to zebrafish i.e., the start from stripe. Even though the sign was opposite to

10 the linear model, total $u$ change may become minus with relation to other components

11 (i.e., $-i_{u v} v-i_{u w} w+d_{u}<0$ in the deformed reaction terms $\left(-i_{u v} v-i_{u w} w+d_{u}\right) u+$

$\left.12 s_{u} /(1+u+v)\right)$. Therefore, the self-productivity can be small enough to agree with the low

13 proliferation rate of melanophores. Similar, concerns about the self-productivity of

14 xanthophores mentioned above, $\partial v / \partial t$ in (5) already had a self-productivity by the

15 multiplication term $v w$, regardless of the sign of $d_{v}$ (i.e., $-i_{v u} u+p_{v w} w-d_{v}>0$ in

16 deformed reaction terms $\left.\left(-i_{v u} u+p_{v w} w-d_{v}\right) v+s_{v} /(1+u+v)\right)$. The sign of $d_{v}$ can be

17 inverted though the change were not expected to substantially affect pattern

18 characteristics. 
2 the condition where respective gates on $U$ and $V$ cells, and both-gate defects were added

3 (Fig. 5A-C). Again, numerical results consistent with linear model could be obtained from

4 an arbitrary parameter set generating a stripe pattern. Biased pattern shift could also be

5 obtained by simulations, partly corresponding to connexin-mutation experiments. When

6 gates on $U$ cell were deleted, it resulted in a $u$ dot pattern (Fig. 5A). Simultaneous

7 decreases in $p_{v w}$ and $c_{w v}$ by increasing the gate defect on $V$ cells generated a net of $u$,

8 though not so drastic (Fig. 5B). Defect in gap-junction by decreasing $i_{v u}$ and $i_{u v}$, had

9 minimal effect, though the stripe region became recessive with combination of defects on

10 each outer gate (Fig. 5C, D). The thinning of the $u$ population was not clear because of the

11 thin stripe at the start. 
3 determining fish pigmentation patterns, channels on pigment cells were generalized as

4 "gates" (Fig. 1). The three-component RD model (1) was shown to be composed of two-

$5 \quad$ variable RD systems bringing diffusion driven instabilities (Fig. 2, 3). The proposed

6 qualitative models help to understand the relationship between pigment cells, as well as

7 between cells and molecules, even when their identity is unknown. The terms of the

8 theoretical model were connected with the functions of each channel on different cells.

9 Parameters affected by the gate defects were deduced (Fig.1G) then the effects of such

10 defects were simulated from a parameter set that generated an arbitrary stripe as a

11 benchmark (Fig. 4, 5). Then, dots and thinning of the $U$ cell population could be obtained

12 by a defect of the gate on it in numerical simulations. The identities of melanophores and

13 xanthophores were deduced from the change of pattern selection and the wavelengths,

14 though the identify of important substance $W$ is still missing. Then, improvements to the

15 nonlinear model enabled a description of the detailed behaviors of components that are

16 related to the pattern formation. Though the numerical analyses could not strictly explain

17 the pattern obtained by experimental manipulation of connexins by Usui et al. (2019), ${ }^{9}$ the 
1 present study can help to interpret the mechanism underlying the leopard mutation, as a

2 Turing system.

4 The signs of $d_{u}$ prefer to invert for a desirable range of pattern selection start from stripe in

5 the improved nonlinear model, though the total changes for $U$ (= melanophore)

6 corresponded to experimental observations. The increase in existing melanophores

7 brought by positive $d_{u}$ were visible by the laser ablation of adjacent xanthophores or

8 deletion of stripes in the experiments in Nakamasu et al. (2009), ${ }^{13}$ Each manipulation

9 decreased $-i_{u v} v u$ or $-i_{u w} w u$ inhibitions, respectively. On the other hand, the self-

10 productivity of xanthophores in the nonlinear model can be achieved even though the

11 negative coefficient of self-reaction. The combination of two-cases of diffusion driven

12 instability in the three-component model indicates the capacity to make a pattern without

13 melanophore by the self-productivity of xanthophore, if sufficient $W$ is added externally.

14 On the other hand, if melanophores have strong self-productivity, it will also be able to make a pattern without xanthophores by the removal of extra $W$. Connexins are related to both hemi-channels and gap junctions. Hemi-channels

17 have been considered less important in physiology, although it was recently revealed that 18 the aberrant activity of hemi-channels could change the proportion of vertebrae ${ }^{34}$ and was 
1 related to the pigment-pattern formation in zebrafish ${ }^{33,35}$ Laser ablation experiments

2 showed that the interactions between xanthophores and melanophores differed depending

3 on the distance. In the present model, the highly diffusible molecule $W$ had a positive

4 effect on $V$. Hence, the inhibition of $V$ via gap junctions is inconsistent. Outflow of harmful

$5 \quad W$ from $U$ via gap junctions is also inconsistent with the inhibition. This elicits a different

6 signal transduction cascade as cell depolarization ${ }^{39}$ and incorporation of functions for

7 molecules other than connexin should be envisioned. Then the inverted function may be

8 derived from an observed rectified current in gap junction. It was observed that quail

9 melanocytes interacted with each other via filopodia in vivo and in vitro. ${ }^{40}$ Gap junctions

10 may have functions on interactions not only between other types of cells but among the

11 same populations. Though, the obtained simulation results included several collisions with

12 experimental results. Similar discords were also in experiments. The results of Usui et al.

$13(2019)^{9}$ indicated that either $\mathrm{C} \times 41.8$ or $\mathrm{C} \times 39.4$ is needed on melanophores then $\mathrm{C} \times 41.8$ is

14 necessary and sufficient on xanthophore for (stripe) pattern formation. However, such

15 "necessary and sufficient" trait for aberrant currents could be observed in hemichannels

16 composed of Cx39.4 in the absence of calcium, in electro-physiological experiments. ${ }^{33}$

17 Shuffle of mutant fish further supported the numerical results shown in Fig. 6. In this case, 
1 expected Cx41.8 manipulations showed the "necessary and sufficient" trait on

2 melanophores.

4 observed both on the body and fins. Because of the fins lack iridophores, the effect on the

5 pigment pattern formation depends on the relationship between melanophores and

6 xanthophores. Even though pattern formation is achieved by the two types of cells, details

$7 \quad$ on the role of iridophores in cellular interactions have been revealed. ${ }^{11}$ The evaluation of

8 the iridophore function in similar modeling will also be possible and should be attempted.

10 mathematical analyses. For example, pattern size was mathematically analyzed with

11 regard the model as combination of two-variable systems here. This method cannot yet

12 describe the independent pattern sizes of each type of pigment cell that have been

13 observed experimentally ${ }^{22}$ and predicted numerically. Therefore, more sophisticated

14 analyses will be required in the future. 


\section{Numerical simulations}

For the linear model, $d_{u}$ was increased from 0 to 0.2 within limits along the $x$-axis

4 (Fig. 4A, D-H) and the parameters $p_{v w}$ and $c_{w v}$ were decreased linearly from 1 to 0 (Fig.

$54 \mathrm{~B}, \mathrm{D}-\mathrm{H})$ for investigation of the effect of gates on each cell. For short-range effects, $i_{u v}$

6 and $i_{v u}$ were decreased linearly from 1 to 0.6 in Fig. 4C. Accordingly, the arbitrary

7 parameter set generating stripes (wild-type) was placed in the right top of the phase plane

8 (Fig. 4D-I). Partial differential equations were calculated with 20000 and 40000 iterations

9 with $d t=0.1$ and $d t=0.05$, in fields sized $x l=56.25, y l=225$ with $d x=0.75$ and $x l=$

$10 y l=200$ with $d s=0.5$ for Fig. 3A-C and Fig. 3D-H, respectively. In Fig. 4l, to investigate

11 the simultaneous gap-junction effects with hemi-channels $s_{d}(x, y)=1-0.002 \max \{x, y\}$,

$12 u_{d}(x)=0.0004 x$, and $v_{d}(y)=1-0.002 y$ were utilized in the field sized $x=y=300$

$13(d x=0.75)$ and $d t=0.1$ for 20000 iterations.

14 For the non-linear model, the parameters $d_{u}$, and $i_{u v}$ and $i_{v u}$ were decreased

15 linearly from 1 to $0.6 . c_{w v}$ and $p_{v w}$ were decreased linearly from 0.5 to 0.1 in Fig. 5 A-C,

16 and decreased by $s_{d}(x, y)=1-0.002 \max \{x, y\}$ simultaneously in Fig. 5D. Accordingly,

17 the arbitrary parameter set generating stripes (wild-type) was placed in the right top of the

18 phase plane. Partial differential equations were calculated in fields sized $x l=10, y l=40$ 
1 in Fig. 5A-C and $x l=y l=50$ with $d s=0.25$ in Fig. 5D. Then after 500000 iterations

2 calculated with $d t=0.01$, we obtained the result.

3 Calculations were performed in the language Full BASIC with no-diffusion boundary

4 conditions then and results are shown as density plots of $u$. Parameters utilized in this

$5 \quad$ study are summarized in Table 1.

6

7 Quantification of color patterns

8 Color pattern complexity and overall tone were quantified from binarized images using the

9 ImageJ as described in Miyazawa et al. (2010). ${ }^{41}$ Briefly, the pattern simplicity score (PSS)

10 is defined as the area weighted mean isoperimetric quotient of the contours extracted from

11 each image. The overall color tone (OCT) of a pattern is defined and calculated as the ratio

12 of white pixels in the binarized image. Analyzed images were prepared by the quaternary

13 connection of a numerical result $(100 \times 100$ individual fields with periodic boundary

14 conditions) of $u$ in each parameter. 


\section{References}

1. Turing AM. The chemical basis of morphogenesis. Philos Trans R Soc Lond B. 1952; 462: $27-73$.

2. Kondo S, Asai R. A reaction-diffusion wave on the skin of the marine angelfish Pomacanthus. Nature. 1995; 376: 765-768.

3. Johnson SL, Africa D, Horne S, Postlethwait JH. Half-tetrad analysis in zebrafish: Mapping the ros mutation and the centromere of linkage group I. Genetics. 1995a; 139: $1727-1735$.

4. Johnson SL, Africa D, Walker C, Weston JA. Genetic control of adult pigment stripe development in zebrafish. Dev Biol. 1995b; 167: 27-33.

5. Kelsh RN. Genetics and evolution of pigment patterns in fish. Pigment Cell Res. 2004; 17: 326-336.

6. Haffter P, Granato M, Brand M, Mullins MC, Hammerschmidt M, Kane D, Odenthal J, van Eeden FJ, Jiang YJ, Heisenberg CP, Kelsh R N, Furutani-Seiki M, Vogelsang E, Beuchle D, Schach U, Fabian C, Nüsslein-Volhard C. The identification of genes with unique and essential functions in the development of the zebrafish, Danio rerio. Development. 1996; 123: 1-36.

7. Watanabe M, Iwashita M, Ishii M, Kurachi Y, Kawakami A, Kondo S, Okada N. Spot 
pattern of leopard Danio is caused by mutation in the zebrafish connexin41.8 gene.

8. Kumar NM, and Gilula NB. The gap junction communication channel. Cell. 1996; 84: 381-388.

9. Usui Y, Aramaki T, Kondo S, Watanabe M. The minimal gap-junction network among melanophores and xanthophores required for stripe-pattern formation in zebrafish. Development. 2019; 146: dev181065.

10. Volkening A. Linking genotype, cell behavior, and phenotype: multidisciplinary perspectives with a basis in zebrafish patterns. Curr Opin Genet Dev. 2020; 63: 7885.

11. Maderspacher F, Nusslein-Volhard C. Formation of the adult pigment pattern in zebrafish requires leopard and obelix dependent cell interactions. Development. 2003; 130: 3447-3457.

12. Parichy D, Turner J. Temporal and cellular requirements for Fms signal indusing zebrafish adult pigment pattern development. Development. 2003; 130: 817-833.

13. Nakamasu A, Takahashi G, Kanbe A, Kondo S. Interactions between zebrafish pigment cells responsible for the generation of Turing patterns. Proc Natl Acad Sci U S A. 2009; 106: 8429-8434. 
14. Meinhardt $\mathrm{H}$, Gierer A. Application of a theory of biological pattern formation based on lateral inhibition. J Cell Sci. 1974; 15: 132-146.

15. Hamada H, Watanabe M, Lau HE, Nishida T, Hasegawa T, Parichy DM, Kondo S. Involvement of Delta/Notch signaling in zebrafish adult pigment stripe patterning. Development. 2014; 141: 318-324.

16. Watanabe M, Kondo S. Fish pigmentation. Comment on "Local reorganization of xanthophores fine-tunes and colors the striped pattern of zebrafish". Science. 2015;

348: 297.

17. Kondo S. An updated kernel-based Turing model for studying the mechanism of biological pattern formation. J Theor Biol. 2017; 414: 120-127.

18. Bullara DW, De Decker Y. Pigment cell movement is not required for generation of Turing patterns in zebrafish skin. Nat Commun 2015; 6: 6971.

19. Volkening A, Sandstede A. Iridophore as a source of robustness in zebrafish stripes and variability in Danio patterns. Nat Commun. 2018; 9: 3231.

20. Owen JP, Kelsh RN, Yates CA. A quantitative modeling approach to zebrafish pigment pattern formation. Elife. 2020; 9: E52998.

21. Asai R, Kondo S. Zebrafish Leopard gene as a component of the putative reactiondiffusion system. Mech Dev. 1999; 89: 87-92. 
22. Watanabe M, Kondo S. Chenging clothes easily: connexin41.8 regulates skin pattern variation. Pigment Cell and Melanoma Res. 2012; 25: 326-330.

23. Takahashi G, Kondo S. Melanophore in the stripe od adult zebrafish do not have the nature to ather, but disperse when they have the space to move. Pigment Cell and Melanoma Res. 2008; 21: 677-686.

24. Sawada R, Aramaki T, Kondo S. Flexibility of pigment cell behavior permits the robustness of skin pattern formation. Genes Cells. 2018; 23: 537-545.

25. Hirata M, Nakamura K, Kanemaru T, Shibata Y, Kondo S. Pigment cell organization in the hypodermis of zebrafish. Dev Dyn. 2003; 227: 497-503.

26. Hirata M, Nakamura K, Kondo S. Pigment cell distributions in different tissues of the zebrafish, with special reference to the striped pigment pattern. Dev Dyn. 2005; 234: 293-300.

27. Mahalwar P, Walderich B, Singh AP, Nusslein-Volhard C. Local reorganization of xanthophores fine-tunes and colors the striped pattern of zebrafish. Science. 2014; 345: $1362-1364$.

28. Gierer A, Meinhardt H. A theory of biological pattern formation. Kybernetics. 1972; 12: $30-39$.

29. Murray JD. Mathmatical Biology II. Seattle, WA: University of Washington; 1989. 

equations on the square. Proc R Soc A. 1991; 434: 1891.

31. Shoji H, Iwasa Y, Kondo S. Stripes, spots, or reversed spots in two-dimensional Turing systems. J Theor Biol. 2003; 224: 339-350.

32. Miura T, Maini PK. Speed of pattern appearance in reaction-diffusion models: Implications in the pattern formation of limb bud mesenchyme cells. Bull Math Biol. 2004; 66(4): 627-649.

33. Watanabe M, Sawada R, Aramaki T, Skerrett IM, Kondo S. The physiological Dev Boil. 2017; 5: 13. 
36. Yamanaka $\mathrm{H}$, Kondo $\mathrm{S}$. In vitro analysis suggests that difference in cell movement during direct interaction can generate various pigment patterns in vivo. Proc Natl Acad Sci U S A. 2013; 111: 1867-1872.

37. Meinhardt H. In Models of Biological Pattern Formation. London: Academic Press; 1982: Chapter 11. Cell determination.

38. Yamaguchi M, Yoshimoo E, Kondo, S. Pattern regulation in the stripe of zebrafish suggests an underlying dynamic and autonomous mechanism. Proc Natl Acad Sci U S A. 2007; 104(12): 4790-4793.

39. Inaba M, Yamanaka H, Kondo S. Pigment Pattern Formation by Contact-Dependent Depolarization. Science. 2012; 335: 677.

40. Inaba M, Jiang T-X, Liang Y-C, Tsai S, Lai Y-C, Widelitz RB, Chuong CM. Instructive role of melanocytes during pigment pattern formation of the avian skin. Proc Natl Acad Sci U S A. 2019; 116: 6884-6890.

41. Miyazawa S, Okamoto M, Kondo S. Blending of animal colour patterns by hybridization. Nat Commun. 2010; 1071: 1-6. 


\section{Acknowledgments}

2 The author would like to thank Masakatsu Watanabe and Masafumi Inaba for persistent

3 stimulating discussions and important comments, as well as members of the Higaki lab. for

4 providing a suitable environment to concentrate on this investigation. I would also like to

5 thank Editage (www.editage.com) for English language editing and the IROAST

6 Proofreading/ Publication Support Program. This research was supported from Grant-in-Aid

7 for Scientific Research on Innovative Areas (The Japan Society for the Promotion of

8 Science), Periodicity and its modulation in plant No.20H05421 and Research grant from

$9 \quad$ Shimadzu Science Foundation. 
1 Figures and Tables

A

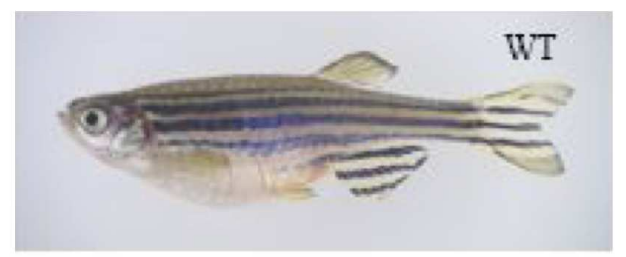

C

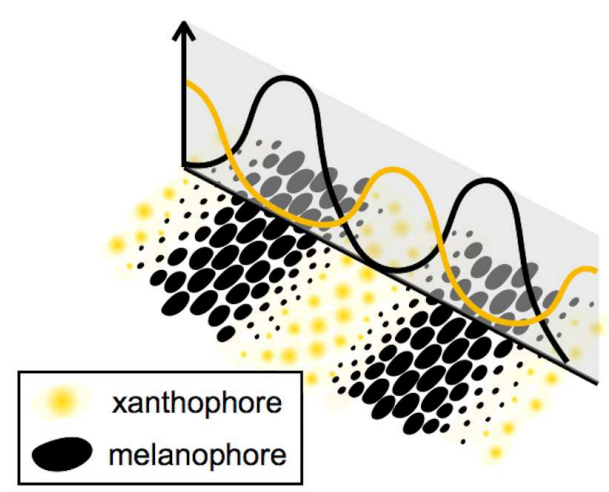

B

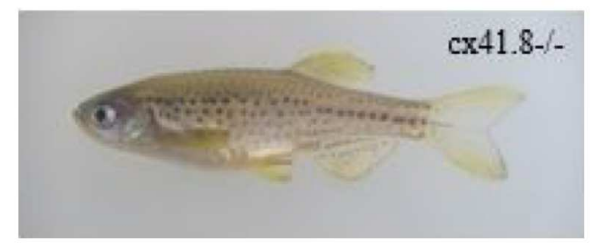

D

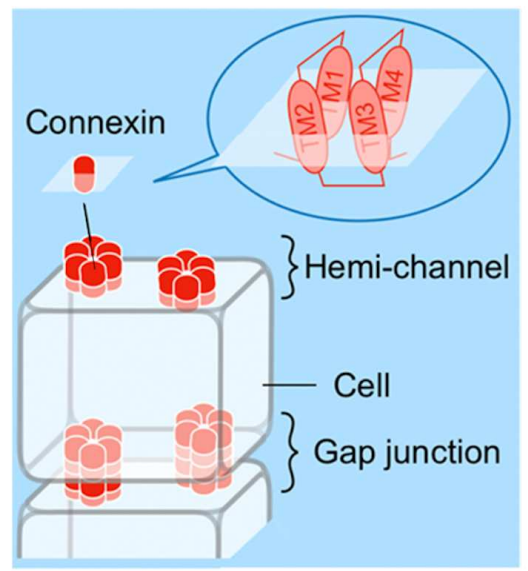

E

\section{Long-range inhibition} for xanthophore

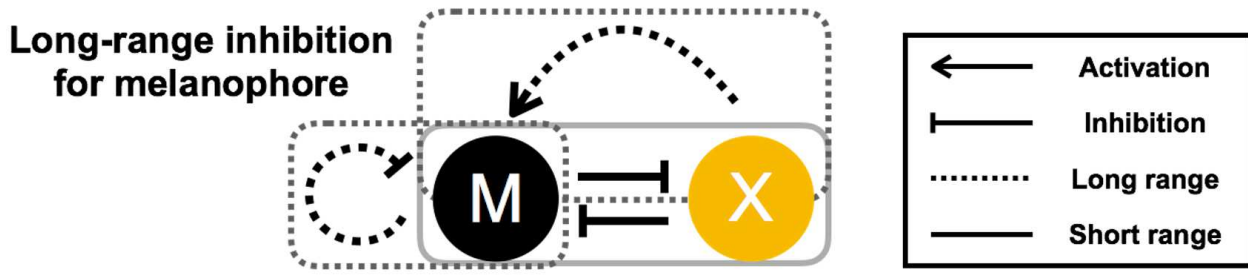

Short-range activation

for each cell

$\mathbf{F}$

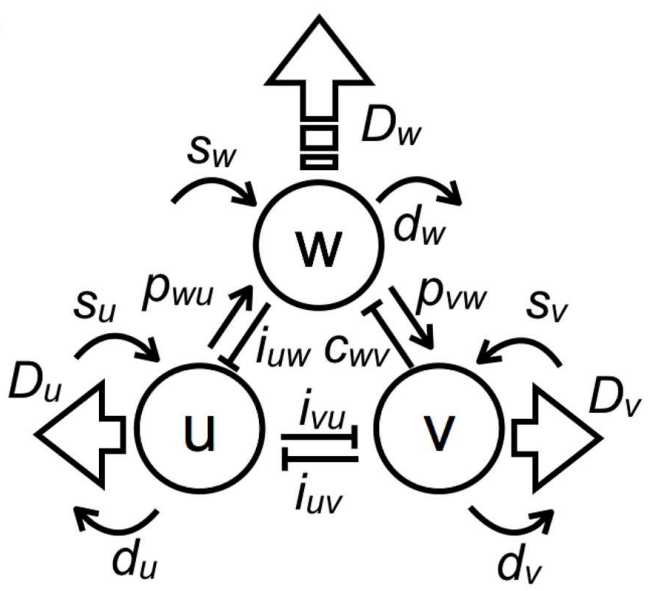

G

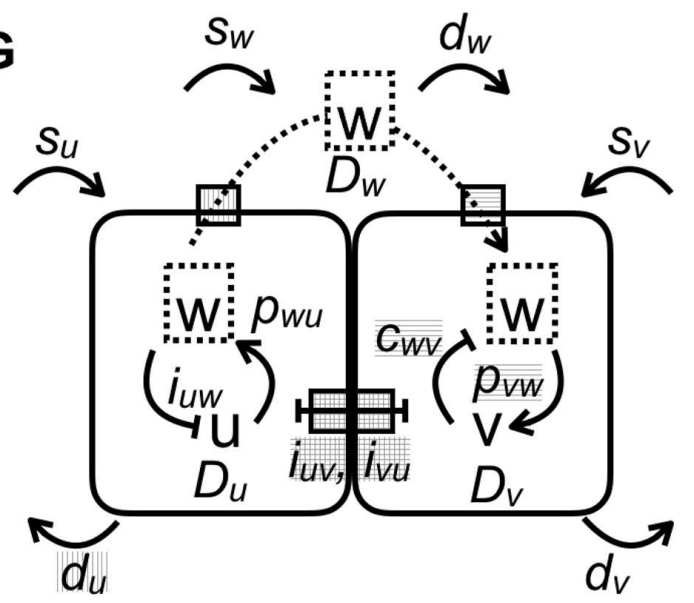

3 Figure 1. Model-based prediction of defects in channels and interactions. 
1 (A) Striped wild-type (WT) zebrafish. (B) Spotted leopard mutant zebrafish. (C) Schematic

2 representation of the relationship between the distribution of pigment cells and the

3 numerical result of the continuous model. (D) Schematic representation of channels

4 composed of connexin complexes. Hemi-channels open to the outside of a cell, whereas

5 gap junctions formed by the docking of hemi-channel connect adjacent cells. (E) Apparent

6 interactions of pigment cells, as revealed by laser ablation experiments. (F) Schematic

7 diagram of a three-variable partial differential equation model composed of components $U$

8 or $V$, which correspond to melanophores or xanthophores, respectively, and component

$9 w$, which represents a highly diffusible molecule. Interactions are indicated by fine arrows,

10 diffusion coefficients (the motility of the components) are indicated by wide arrows, and

11 corresponding parameters are indicated. (G) Schematic diagram of the effect of channels

12 on pigment cells according to the mathematical model. Parameters related to the function

13 of each gate are indicated by hatchings.

14 
A
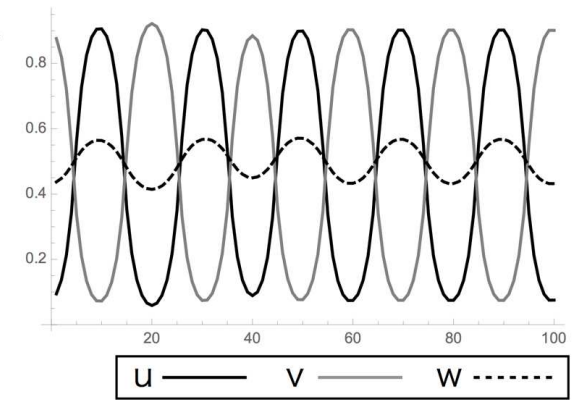

B

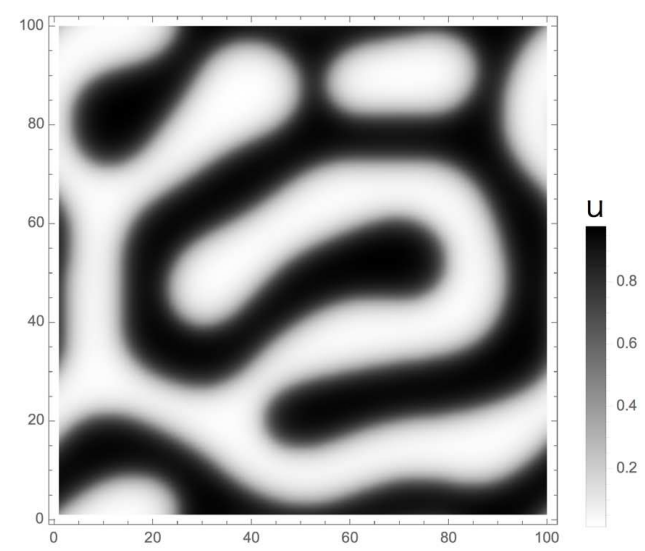

1

$2 \quad$ Figure 2. Turing pattern obtained by applying a reaction-diffusion model.

3 (A), (B) Calculation results of the model had linear terms with limits. (A) One dimension and

4 (B) two dimensions. Results indicate Turing patterns, in which the variables $u$ and $v$ are

5 distributed complementarily and the highly diffusible molecule $W$ peaks at high $u$. 
A

B
Activator-Inhibitor
$\left(\begin{array}{ll}+ & - \\ + & -\end{array}\right)$
Activator-
substrate depletion
$\left(\begin{array}{cc}+ & + \\ - & -\end{array}\right)$

C

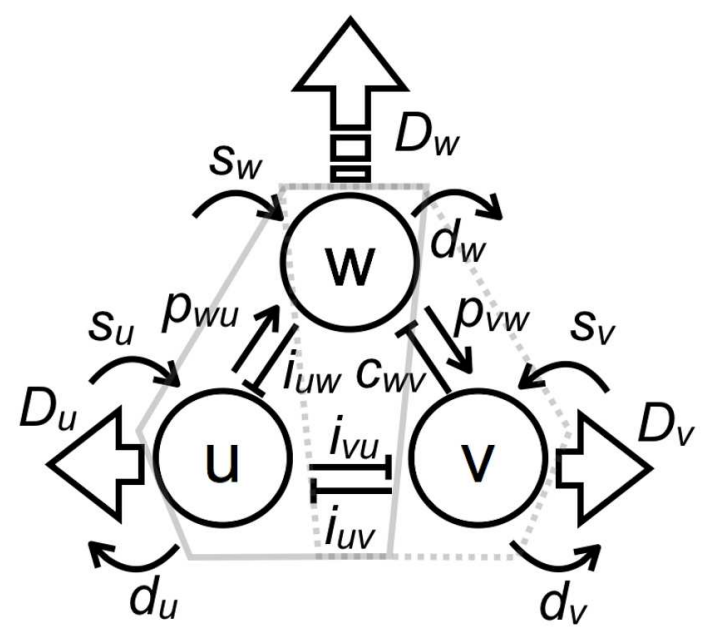

D

$$
\left(\begin{array}{ccc}
-f_{x} & f_{x} & f_{y} \\
f_{x} & -f_{x} & f_{v} \\
g_{x} & g_{x} & g_{y}
\end{array}\right)\left(\begin{array}{c}
u \\
v \\
w
\end{array}\right) \quad\left(\begin{array}{rrr}
-d_{u} & -i_{u v} & -i_{u v} \\
-i_{v u} & -d_{v} & p_{v w} \\
p_{w u} & -c_{w v} & -d_{w}
\end{array}\right)\left(\begin{array}{c}
u \\
v \\
w
\end{array}\right)
$$

2 Figure 3. Parameter correspondences to two-variable systems and effects on pattern

3 size.

4 (A, B) Two conditions that generate a Turing pattern, activator-inhibitor type (A) or

5 activator-substrate-depletion type (B), with different signs of reaction parameters. (C)

6 Correspondence between parameters in the present tree-component model and the two-

$7 \quad$ variable systems. Interactions included in conditions (A) and (B) are enclosed by solid and

8 dashed polygons, respectively. (D) Matrix forms of reaction terms corresponding to the two- 
1 variable system: the filled squares indicate the parameter that increases pattern size when

2 the absolute value is decreased, and the open squares denote the opposite; the solid

3 squares correspond to condition (A), and the dashed ones correspond to condition (B). 

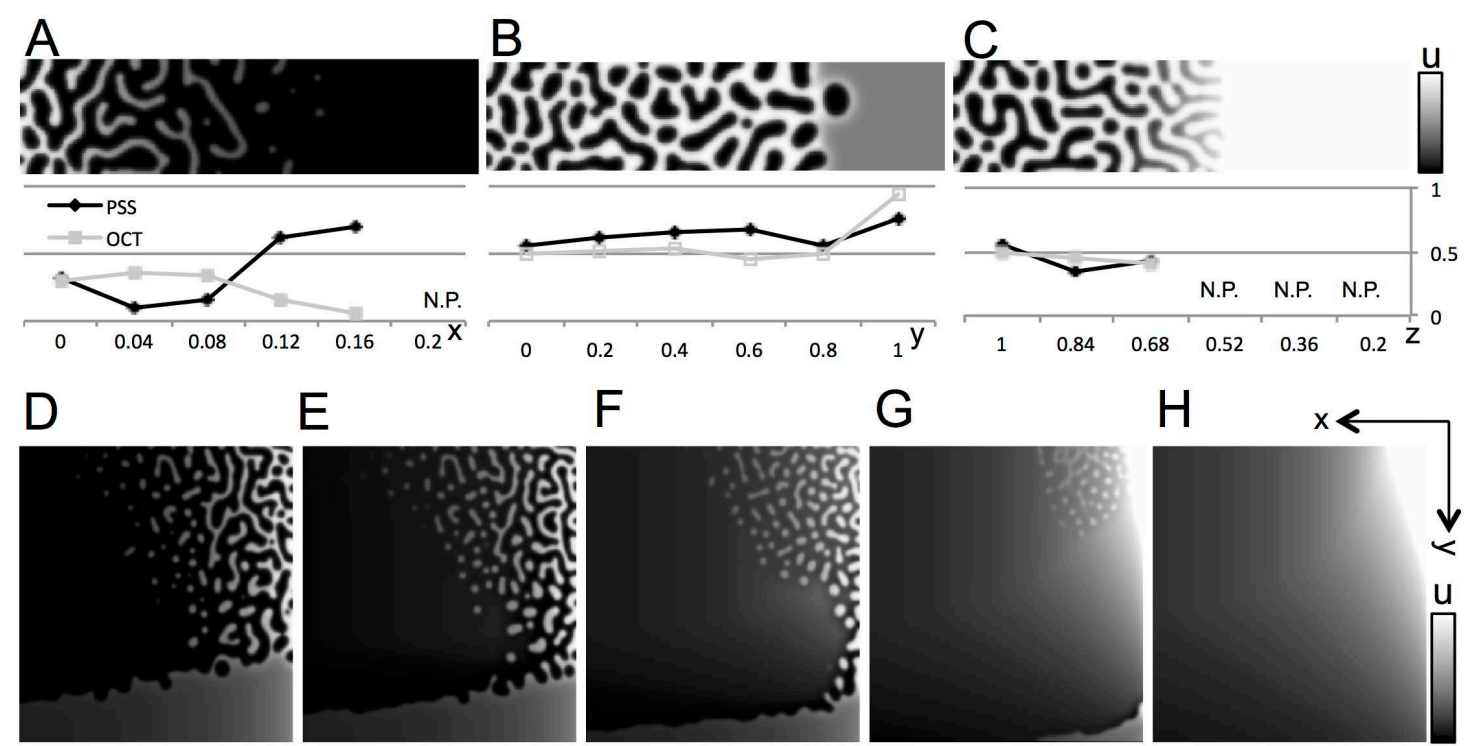

$\mathrm{F}$

G
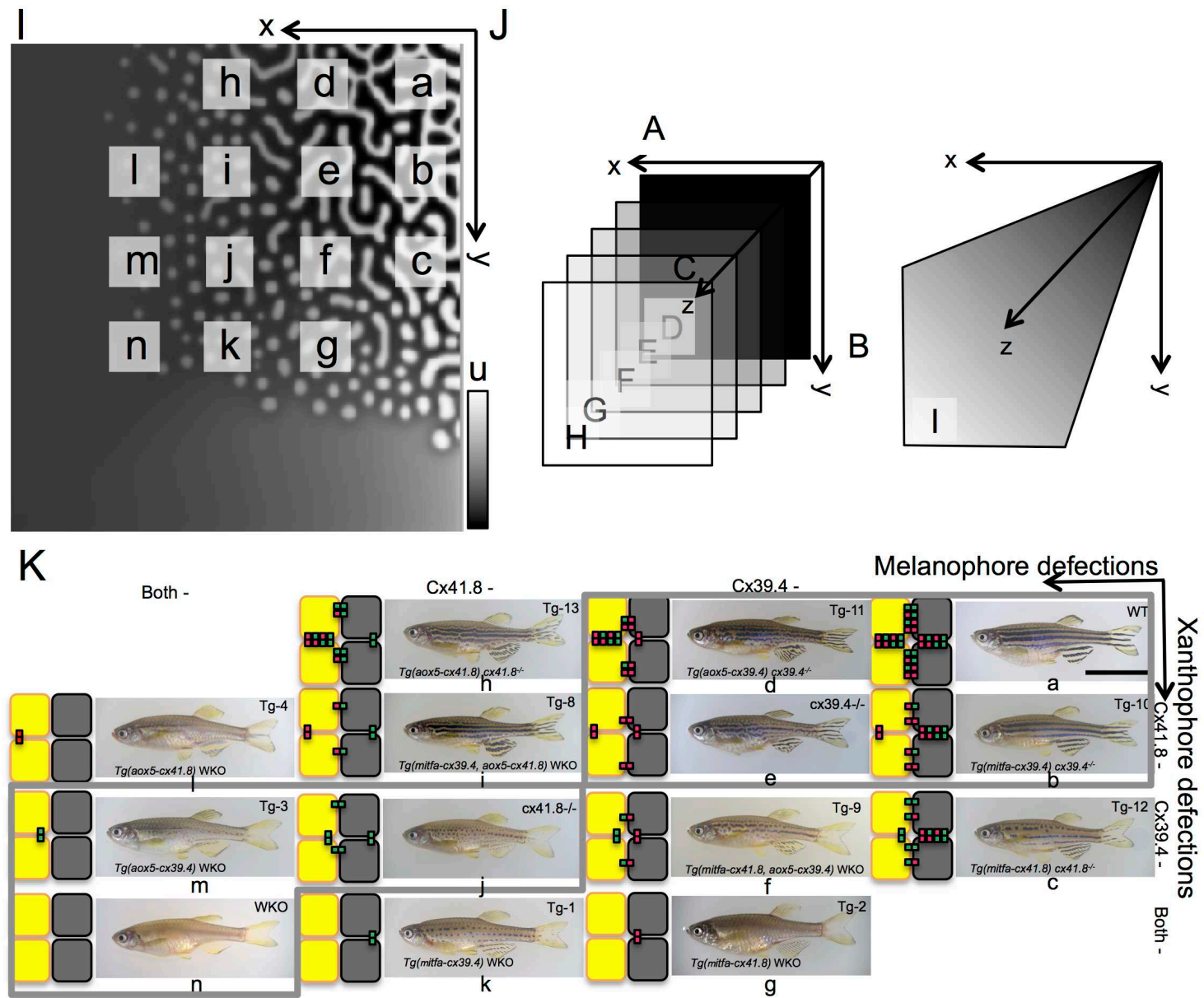

2 Figure 4. Numerical results of gate effects and comparison with experiments 
1 (A)-(I) Numerical results obtained from a model of linear reaction terms with limits. Pattern

2 shifts were observed when the effects of gate defects on both cells were changed. defects

3 of gates on $U$ and $V$ cells, and between the cells, become larger along the $x$-and, $y$-,

4 and $z$ (or $x, y$ )- axes, respectively. (A)-(C) each defect was analyzed independently with

5 quantification of patterns. (A) $U$ defect, (B) $V$ defect, and (C) short-range defects. Pattern

6 simplicity scores (PPS) and overall color tones (OCT) were obtained quantitatively. The

7 short-range effects by gap-junctions were eliminated from arbitral wild-type condition(D)

$8 \quad 0.05$ to $(E) 0.04,(F) 0.03,(G) 0.02$, and $(H) 0.01$. (I) Numerical result of linear terms with

9 limits in which the short-range effects were spontaneously changed along the $x$-and $y$ -

10 axes with increasing $u_{d}$ and decreasing $v_{d}$. Each small letter indicates the corresponding

11 fish with connexin conditions. (J) Schematics of this analyses utilizing the present linear

12 model. $U$ defect, (B) $V$ defect, and (C) short-range defects were changed along with the

13 axes in each panel, as shown in the figures. (K) Pigmentation pattern on connexin-

14 manipulated fish from Usui et al., 2019. Gray flamed fish had correspondence to numerical

15 results.

16 


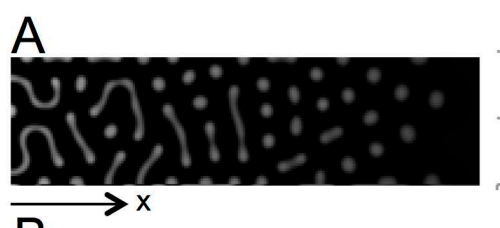

B
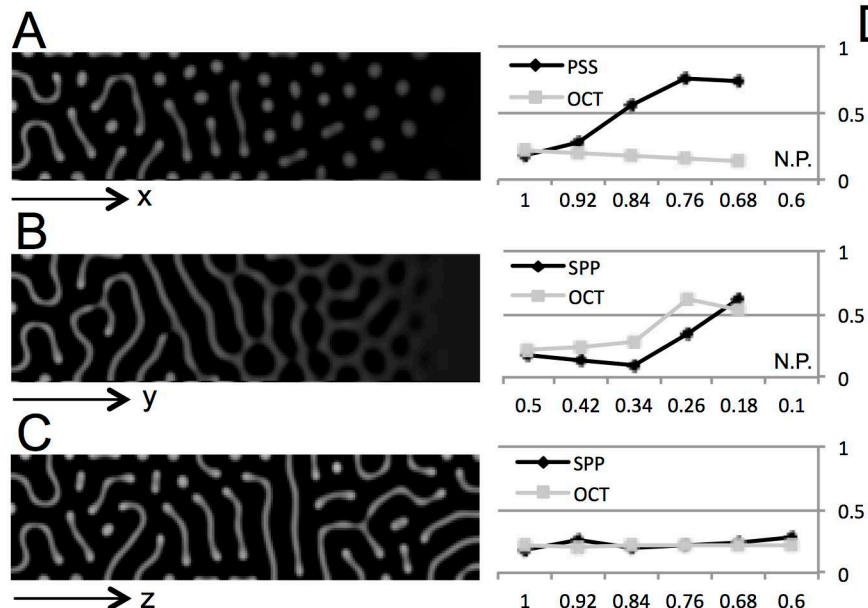

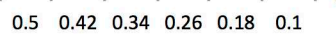

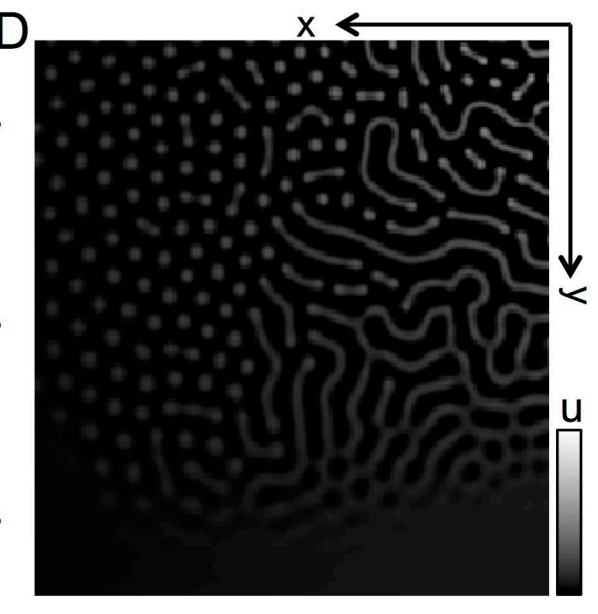

1

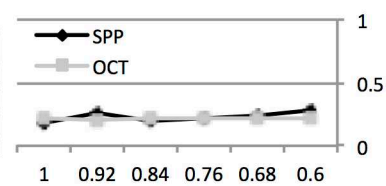

2 Figure 5. Numerical results of gate effects by nonlinear model

3 (A-D) Numerical results obtained from a model with nonlinear reaction terms. Pattern

4 shifts were observed when the effects of gate defects on both cells were changed. (A) $U$

5 defect, (B) $V$ defect, and (C) short-range defects, and between the cells, became larger

6 along the $x$-, $y$-axes, and $z$ (or $x, y$ )- axes, respectively. Pattern quantification by PSS

7 and OCT are shown on the right. Then the short-range inhibitions were decreased along

8 both axes, spontaneously. All results are shown as density plots of $u$. 


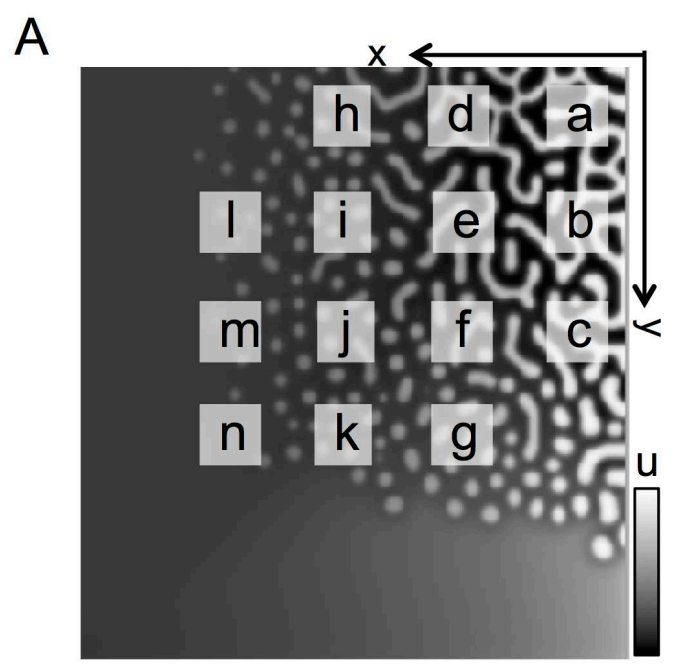

B

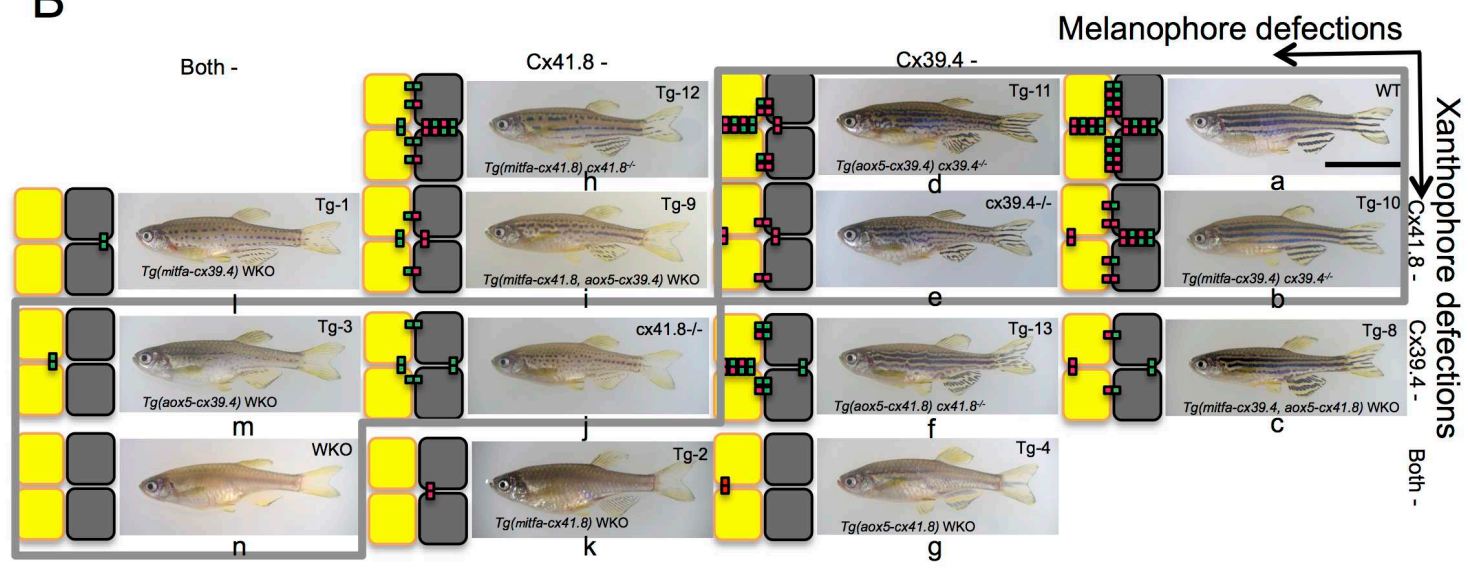

2 Figure 6. Shuffle of mutant fish which gives accordance with numerical results.

3 (A) Density plot of $u$ in calculation results that same as Fig. 4G. The model had linear

4 terms with limits in which the short-range effects were spontaneously changed along the $x$ -

5 and $y$-axes with defects in gates on $u$ and $v$ cells, respectively. (B) Mutant fish were

6 shuffled for accordance with numerical results. Each small letter indicates the

7 corresponding fish with simulation in the aspect of pattern selection. 
1 Table 1. Parameter set utilized in this paper.

\begin{tabular}{|c|c|c|c|c|c|c|c|c|c|c|c|c|c|c|c|c|}
\hline & $D_{u}, D_{v}$ & $d_{u}$ & $i_{u v}$ & $i_{u w}$ & $s_{u}$ & $i_{v u}$ & $d_{v}$ & $p_{v w}$ & $s_{v}$ & $D_{w}$ & $p_{w u}$ & $c_{w v}$ & $d_{w}$ & $s_{w}$ & $\begin{array}{l}\text { min-max } \\
(\text { for } f, g \text { ) }\end{array}$ & $\begin{array}{l}\min -\max \\
(\text { for } h \text { ) }\end{array}$ \\
\hline Fig. 2 & .01 & .01 & .05 & .05 & .05 & .05 & .01 & .05 & 0 & 1 & .05 & .07 & .05 & .02 & $.0-.01$ & $.0-.05$ \\
\hline Fig.4A-D & & & & & & & & & & & & & & & & \\
\hline Fig.4E & .01 & .01 & .04 & .05 & .05 & .04 & .01 & .05 & 0 & 1 & .05 & .07 & .05 & .02 & $.0-.01$ & $.0-.05$ \\
\hline Fig.4F & .01 & .01 & .03 & .05 & .05 & .03 & .01 & .05 & 0 & 1 & .05 & .07 & .05 & .02 & $.0-.01$ & $.0-.05$ \\
\hline Fig.4G & .01 & .01 & .02 & .05 & .05 & .02 & .01 & .05 & 0 & 1 & .05 & .07 & .05 & .02 & $.0-.01$ & $.0-.05$ \\
\hline Fig. $4 \mathrm{H}$ & .01 & .01 & .01 & .05 & .05 & .01 & .01 & .05 & 0 & 1 & .05 & .07 & .05 & .02 & $.0-.01$ & $.0-.05$ \\
\hline Fig.5A-D & .01 & +)1. & 1. & 1. & .2 & 1. & .1 & 1. & .5 & 1 & 1 & 1. & 1. & .0 & - & - \\
\hline
\end{tabular}

